\title{
Motor-cortical activity in tetraplegics
}

\author{
It may eventually be feasible to reconstruct voluntary motor activity in the paralysed.
}

P aralysed patients may benefit from the development of an implantable brain-computer interface device that can bypass damaged motor pathways ${ }^{1-3}$. But it is unclear whether chronically de-efferented areas will still be sufficiently excitable to respond to motor attempts ${ }^{4,5}$ if the motor cortex has been extensively reorganized ${ }^{6,7}$, and, if they are, whether this excitability is somatotopically organized ${ }^{8,9}$. Here we use functional magnetic resonance imaging to study brain activity in subjects with spinalcord injuries while they are executing, or attempting to execute, movements of different limbs. We show that their motor-cortical activation closely follows normal somatotopic organization in the primary and non-primary sensorimotor areas. Our results indicate that any reorganization of the motor system that does occur in these patients does not affect attempt-related activation, and that it should be possible to access voluntary control signals by using a cortical neuroprosthetic.

We examined five subjects with spinalcord damage (C5-C6 trauma-related injuries, $1-5$ years after injury; mean age, $28.4 \mathrm{yr}$ ) who were asked to repetitively move, or attempt to move, their hands (adduction-abduction of the fingers), elbows (flexion), feet (toe flexion), left knee (extension) and lips (pursing) during alternating 30-s blocks. Subjects were instructed to attempt movements they were unable to make, rather than to imagine them, and they reported being able to follow these instructions. Their ability to execute the different movements was evaluated visually and by using surface electromyographic measurements.

One subject had residual use of his hands, whereas the other four, when attempting to abduct and adduct their fingers, elicited fasciculations of muscles in the wrist and palm. All five were unable to move their legs voluntarily. To assess the somatotopy of cortical activation, we used surface projections of statistical parametric maps ${ }^{10}$. Group comparisons were made with a healthy control group ( $n=5$; mean age, $28.0 \mathrm{yr}$ ) by using surface renderings of 'group brains' (group averages of spatially normalized brains). As the pre- and post-central gyri of the individual brains extensively overlapped, they were also visible in the group surface renderings, including a 'knob' that marked the hand motor representation ${ }^{11}$.

Figure 1 shows that activation was significant in the injured group when attempting hand movements (four subjects) on the two banks of the contralateral central sulcus (primary motor and sensory cortices), extending towards the pre- and post-central sulci (pre-

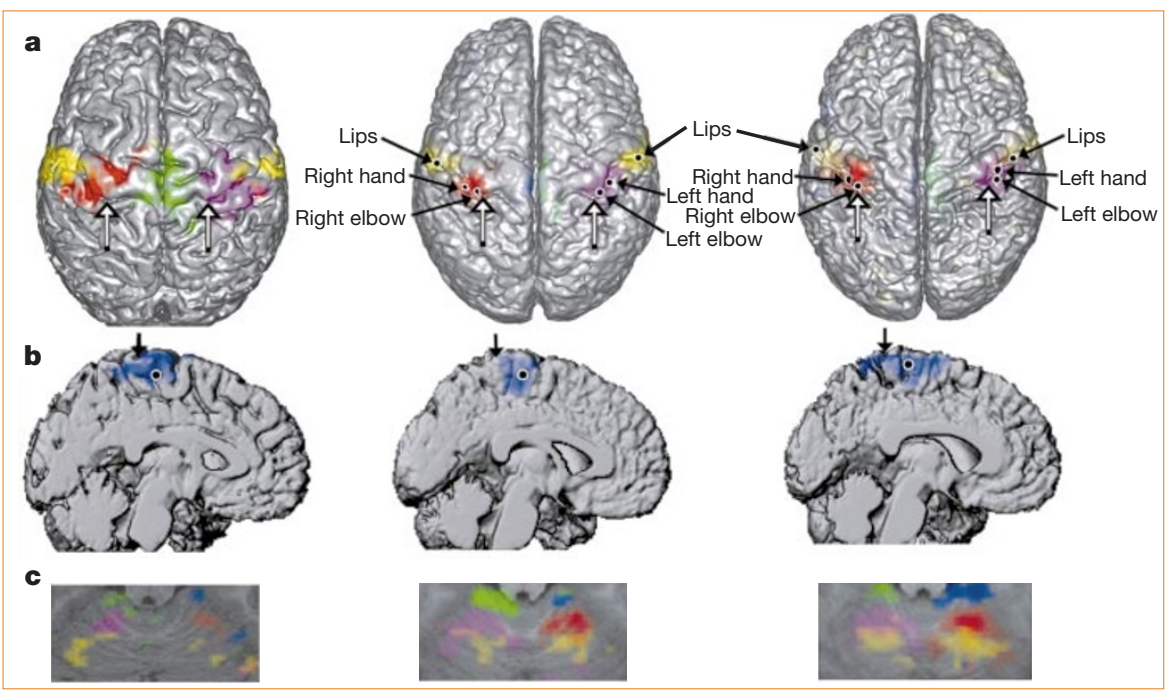

Figure 1 Cortical and cerebellar activation associated with attempted and executed movements in a paralysed individual (left), paralysed group (middle) and control group (right). a, b, Scaled integrals of $t$-test statistics ( $1.2 \mathrm{~cm}$ beneath the cortical surface); black dots indicate the location of $t$-value maxima. In a (top view), blue areas of activation are omitted from the left-side panel to avoid overlap; hollow arrows, putative hand-notches on the precentral gyri; $\mathbf{b}$, mid-sagittal renderings of left-hemisphere activation resulting from attempted flexion of right toes. Arrows, central sulcus. c, Activation maps (SPM[t\}) in axial sections through the anterior cerebellum (21 mm inferior to the anterior-posterior commissural line). Red, abduction-adduction of fingers on the right hand, and purple, left hand; blue, flexion of right toes, and green, left toes; yellow, lip pursing.

motor and parietal regions). The attempted toe flexion and knee extension (five subjects) resulted in bilateral activation (stronger on the contralateral side) along the medial aspect of the longitudinal fissure (extending along the entire paracentral lobule). Executed movements of the elbows (results not shown) gave activation patterns that overlapped the one observed during attempted hand movements (elbow activation patterns were more dorso-posterior). Lip pursing resulted in activation on the most lateral aspect of the sensorimotor cortex.

Further consistent, organized activation occurred in the anterior cerebellum (Fig. 1c), the dorsal cingulate motor area, and an area extending on the banks of the sylvian fissure from the central sulcus (SII) to the operculum and the insula (results not shown). All of these activation features were reproduced in the control group (Fig. 1b, right panel) and are consistent with previous studies of cortical ${ }^{12}$ and cerebellar ${ }^{13}$ motor maps. Although a consistent dorso-posterior shift of hand and elbow activation maxima was evident between injured and control groups, a similar shift was seen in the location of the group hand-knob, indicating that it may be due to intergroup anatomical differences.

We have shown that volitional activation of cortical sensorimotor representations in sufferers of paralysis occurs with only minimal reorganization of the gross somatotopy in subjects within five years of spinal-cord injury. The remnant motor representations in these subjects seem to respond to intent, one of the principal physiological requirements for the development of a braincomputer interface device. Rapid progress in neural-interface technology may soon enable implantable neuroprosthetics to provide real-time reconstruction of complex voluntary motor activity for the paralysed.

Shy Shoham*, Eric Halgren $\dagger$,

Edwin M. Maynard ${ }^{\star}$, Richard A. Normann* ${ }^{*}$ Department of Bioengineering, University of Utah, Salt Lake City, Utah 84112, USA

e-mail:normann@utah.edu

$\dagger$ Nuclear Magnetic Resonance Center,

Massachusetts General Hospital, Charlestown,

Massachusetts 02129, USA

1. Kennedy, P. R., Bakay, R. A. E., Moore, M. M., Adams, K. \& Goldwaithe, J. IEEE Trans. Rehab. Eng. 8, 198-202 (2000).

2. Wessberg, J. et al. Nature 408, 361-365 (2000).

3. Nicolelis, M. A. L. Nature 409, 403-407 (2001).

4. Green, J. B., Sora, E., Bialy, Y., Ricamato, A. \& Thatcher, R. W. Neurology 53, 736-743 (1999).

5. Lotze, M., Laubis-Hermann, U., Topka, H., Erb, M. \& Grodd, W. Restor. Neurol. Neurosci. 14, 183-187 (1999)

6. Sanes, J. N. \& Donoghue, J. P. Annu. Rev. Neurosci. 23, 393-415 (2000).

7. Raineteau, O. \& Schwab, M. E. Nature Rev. Neurosci. 2, 263-273 (2001)

8. Cheney, P. D. et al. Prog. Brain Res. 128, 135-160 (2000).

9. Turner, J. A. et al. IEEE Trans. Neural Syst. Rehab. Eng. 9, 154-160 (2001).

10. Friston, K. J. et al. Hum. Brain Mapping 2, 189-210 (1995).

11. Yousry, T. A. et al. Brain 120, 141-157 (1997)

12. Fink, G. R., Frackowiak, R. S., Pietrzyk, U. \& Passingham, R. E. J. Neurophysiol. 77, 2164-2174 (1997).

13. Nitschke, M. F., Kleinschmidt, A., Wessel, K. \& Frahm, J. Brain 119, 1023-1029 (1996). 